\title{
Marina Gasnier et Pierre Lamard dir., Le patrimoine industriel comme vecteur de reconquête économique
}

Collection Histoire, Mémoire, Patrimoine, UTBM, Editions Lavauzelle, 2007

\section{Gracia Dorel-Ferré}

\section{OpenEdition}

\section{Journals}

Édition électronique

URL : http://journals.openedition.org/dht/319

DOI : $10.4000 /$ dht.319

ISSN : 1775-4194

Éditeur :

Centre d'histoire des techniques et de l'environnement du Cnam (CDHTE-Cnam), Société des élèves du CDHTE-Cnam

\section{Édition imprimée}

Date de publication : 1 décembre 2009

Pagination : 225-226

ISBN : 978-2-9530779-4-0

ISSN : 0417-8726

Référence électronique

Gracia Dorel-Ferré, « Marina Gasnier et Pierre Lamard dir., Le patrimoine industriel comme vecteur de reconquête économique», Documents pour l'histoire des techniques [En ligne], 18| 2e semestre 2009, mis en ligne le 24 septembre 2010, consulté le 24 septembre 2020. URL : http:// journals.openedition.org/dht/319; DOI : https://doi.org/10.4000/dht.319

Ce document a été généré automatiquement le 24 septembre 2020.

(c) Tous droits réservés 


\section{Marina Gasnier et Pierre Lamard dir., Le patrimoine industriel comme vecteur de reconquête économique}

Collection Histoire, Mémoire, Patrimoine, UTBM, Editions Lavauzelle, 2007

Gracia Dorel-Ferré

\section{RÉFÉRENCE}

Marina Gasnier et Pierre Lamard dir., Le patrimoine industriel comme vecteur de reconquête économique, Collection Histoire, Mémoire, Patrimoine, UTBM, Editions Lavauzelle, 2007. 
1 En 2006, dans la même collection, Pierre Lamard, en partenariat, cette fois avec Marie-Claire Viotoux, mais déjà en collaboration avec Marina Gasnier, avait dirigé un ouvrage sur Les friches industrielles, point d'ancrage de la modernité. Ce présent volume fait suite et le complète. Si ce compte rendu traite du second, il garde le premier en référence constante et le lecteur tirera le plus grand profit à consulter les deux. Ils sont la contribution des universitaires à un sujet qui jusqu'alors avait surtout été l'apanage des géographes, des économistes ou des aménageurs. Il s'agissait d'analyser les effets de la désindustrialisation, évaluer les mises en friches, mettre en évidence les procédures de patrimonialisation. Les

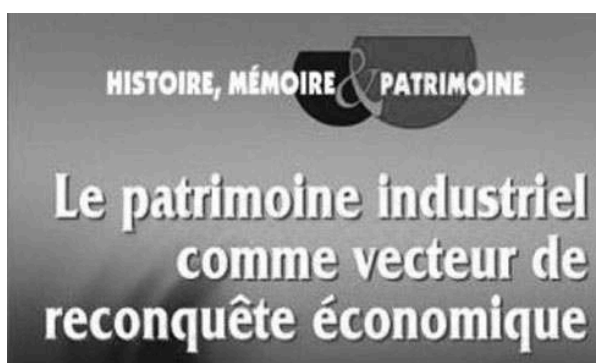

Marina Gasnier et Pierre Lamard (dir.)

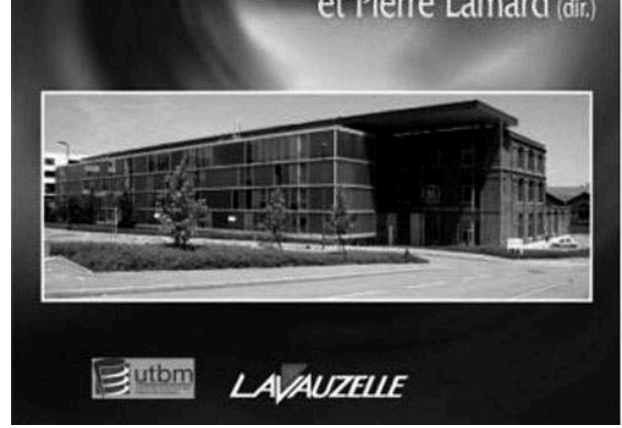
auteurs ont choisi, à travers des communications variées, de croiser les approches tout en maintenant le cap sur ce qui leur semblait être une promesse d'avenir : les deux ouvrages, et tout particulièrement Le patrimoine industriel comme vecteur de reconquête économique, sont aussi un encouragement et un message d'espoir.

Il est vrai que les choses ont changé : on ne regarde plus les friches industrielles comme une malédiction, mais comme des réserves patrimoniales que l'imagination, autant que les subventions, permettront de réaffecter. Pour ce changement de regard, il a fallu laisser s'écouler une trentaine d'années, semées de destructions irréversibles ou d'abandons irréparables. Les questions sont loin d'être résolues cependant.

Dans un Dossier pour l'Art, consacré aux friches industrielles et publié par le Centre national de Documentation Pédagogique d'alors, en 1976, on évoquait déjà la difficulté, pour ne pas dire l'impossibilité, de mettre en valeur et réaffecter un bâtiment industriel d'architecture très finalisée: des salles d'une manufacture de tabacs ou d'une salle de tissage peuvent être reconverties à d'autres activités mais que faire d'un haut-fourneau sinon un musée? Que faire des silos ou des grands moulins sinon les évider et garder, éventuellement, la carcasse ? Autre difficulté : une friche excentrée, difficile d'accès a toutes chances d'être irrémédiablement abandonnée. Enfin, qui dit réutiliser, réaffecter, ne dit aucunement garder le sens de l'édifice antérieur. Et, il faut bien le reconnaître, la plupart des bâtiments qui ont été sauvés de la destruction, l'ont été au détriment de leur identité. Il est rare que l'on se préoccupe d'évoquer l'usine : on se contente d'utiliser des volumes intéressants, pour d'autres fonctions, c'est tout. Ainsi, dès la formation même de ces friches industrielles, on savait que le combat serait difficile, et que la société civile serait dure à convaincre. Les exemples étudiés dans l'ouvrage de Pierre Lamard et Marina Gasnier nous donnent une typologie d'initiatives qui sans être des réussites ont permis à des villes de faire du neuf avec du vieux, depuis la mise en musée, la réutilisation multifonctionnelle, la réhabilitation industrielle. Deux faits récents, en ce qui concerne l'espace français, semblent y avoir contribué : les 
réaménagements des centres-villes et la nouvelle structuration institutionnelle en communautés de communes.

4 Le réaménagement des centres-villes a permis de poser la question du devenir des friches autrement que par l'arasement, comme ce fut le cas pour les espaces miniers ou métallurgiques de la Lorraine désindustrialisée. On ne pouvait, pour les centres-villes adopter une méthode aussi brutale, et l'on sait le rôle pionnier tenu par des architectes comme Reichen et Robert dans la requalification de friches telles que l'usine Le Blan à Lille ou l'usine Blin et Blin à Elbeuf. Une dimension internationale autre que la seule référence à l'Emscher Park, nous aurait confortés dans cette direction. Un récent colloque à Aguascalientes (Mexique) permettait de voir le parti que l'on tirait d'une friche de 92 ha au cœur même de la ville, qui a choisi de transformer les espaces des ateliers de réparation des chemins de fer, devenus inutiles, en un complexe multifonctionnel de salles de conférences, école de musique et de danse, musée du chemin de fer, bureaux, université, parc public, etc. Il s'agit d'une opération de requalification urbaine menée par la ville mais suivant des marchés offerts aux entreprises privées. Il s'agit d'offrir aux habitants une suite de services dont ils manquaient jusque-là. Le résultat est particulièrement spectaculaire. Les exemples analysés dans le volume, en particulier ceux qui concernent Nantes et la région lyonnaise, montrent qu'aujourd'hui une grande variété d'usages préside à la réaffectation des friches industrielles. Remarquons en passant que cette typologie dans le réemploi n'a rien de spécifique: les friches militaires sont réutilisées de la même manière, et Bernard André souligne à juste titre la concurrence qu'elles exercent à l'heure des choix financiers.

5 Les donneurs d'ordre sont nouveaux aussi, en France du moins, avec les nouvelles communautés de communes. Celles-ci se sentent les reins plus solides pour entreprendre des projets un peu ambitieux, en général tournés vers l'exploitation touristique. Cette dimension n'est pas à dédaigner. Certes, comme le souligne Bernard André, la vocation du patrimoine industriel ne réside pas uniquement dans une mise en circuit touristique. Mais les réussites dans ce domaine montrent qu'il y a là des perspectives non seulement rentables mais aussi pédagogiques.

6 C'est d'ailleurs sur un volet pédagogique que se termine l'ouvrage, avec le récit d'expériences menées à l'université de Sévenans. Ainsi le puits Arthur de Buyer est réhabilité en centrale géothermique et le filature de laine peignée de Malmerspach en espace de loisirs. Bien que rejetés à la fin de l'ouvrage de tels exercices sont fondamentaux. Marina Gasnier, dans son introduction, déplore que la question des friches patrimoniales soit absente des programmes scolaires. Effectivement, à part un moment du programme de géographie, dans les années 1970, cette question n'est pour ainsi dire pas abordée. Or, si pour la société civile, la réaffectation d'une chapelle désaffectée ne pose pas de problème, c'est bien parce que dès le plus jeune âge nous apprenons à considérer le patrimoine religieux comme quelque chose d'important et de respectable. Nul doute que notre approche du patrimoine industriel serait tout autre si dès l'école élémentaire, comme nous l'avons toujours recommandé, on apprenait à repérer et à comprendre les éléments du patrimoine de l'industrie qui se trouvent autour de nous.

7 Le patrimoine industriel comme vecteur de reconquête économique, associé à l'ouvrage qui l'a précédé, constitue un bon diagnostic et un panel bien choisi d'expériences, surtout 
hexagonales. Il est une bonne approche au sujet, appuyé sur une réflexion théorique solide et documentée, avec laquelle il faudra compter désormais.

\section{AUTEURS}

\section{GRACIA DOREL-FERRE}

CDHTE-Cnam 